\title{
Root-MUSIC Time Delay Estimation Based on Propagator Method
}

\author{
Bin $\mathrm{Ba}$, Yun Long Wang, Na E Zheng \& Han Ying Hu
}

Zhengzhou Institute of Information Science and Technology, Zhengzhou, 450002, China

\begin{abstract}
Keywords: time delay estimation, Root-MUSIC, propagator method, computational complexity.
\end{abstract}
\begin{abstract}
This paper presents a new root multiple signal classification (Root-MUSIC) time delay estimation method based on the concept of well known propagator method (PM). The PM does not need the eigenvalue decomposition whose computational complexity is high. This method has great performance of time delay estimation under the condition of high signal to noise ratio (SNR) and need spectral peak searching whose computational complexity is very high. To address those issues, the new method is proposed. The proposed algorithm has high resolution capabilities and has lower computational complexity than conventional PM. Its computational complexity significantly reduces by using the method of finding the root of polynomial instead of the spectral peak searching and the eigenvalue decomposition. Theoretical analysis and computer simulation results show that the proposed method is effective.
\end{abstract}

\section{Introduction}

Positioning refers to the geographic information of a target. Various needs from commercial and emergency promote the improvement of high accuracy, low cost positioning system. Location information is very helpful for improving performance of the communication system.

The super resolution techniques are firstly used in direction of arrival (DOA) estimation. The DOA estimation has many applications in wireless localization [1-6]. Moreover, the super resolution time delay estimation can be used in high-precision ranging, which is the basis of highprecision positioning system. The multiple signal classification (MUSIC) is used to estimate time delay [7-9]. Smoothing in frequency domain is introduced to MUSIC algorithm for single snapshot. The MUSIC algorithm has great performance in time delay estimation. However, it needs eigenvalue decomposition and spectral peak searching whose computational complexity is very high. To address this issue, the estimation of signal parameters via rotational invariance technique (ESPRIT) is provided to time delay estimation [10]. It does not need spectral peak searching and its computational complexity significantly reduces. However, the performance of ESPRIT is lower than MUSIC. In order to avoid spectral peak searching and decline for performance, the RootMUSIC is introduced to time delay estimation. The spectral peak searching is replaced by finding the roots of polynomial [11]. The Root-MUSIC and MUSIC have the same asymptotic performance, and the Root-MUSIC performance with small samples is better than the MUSIC. However, the Root-MUSIC algorithm needs eigenvalue decomposition, whose computational complexity needs to reduce. The propagator method (PM) can estimate noise subspace without eigenvalue decomposition [12].

In this paper, a new Root-MUSIC time delay estimation algorithm based on PM (PM-RootMUSIC) is proposed. The algorithm does not need eigenvalue decomposition and spectral peak searching, so its computational complexity significantly reduces.

\section{Signal model}

The impulse response of a multipath wireless channel in time domain can be modelled as

$$
h(t)=\sum_{k=0}^{L_{p}-1} a_{k} \delta\left(t-\tau_{k}\right),
$$

where $L_{P}$ is the number of multipath components, $a_{k}=\left|a_{k}\right| e^{j \theta_{k}}$ and $\tau_{k}$ are the complex fading coefficient and propagation time delay for the $k$ th path component, respectively. $\left|a_{k}\right|$ presents the 
amplitude of $a_{k}$. $\theta_{k}$ means the phase of $a_{k}$ and obeys a uniform distribution $U(0,2 \pi)$. In consequence, $\tau_{0}$ denotes the propagation time delay of the first path. This time delay has to be estimated for the purpose of locating a target.

The time domain expression of OFDM symbol is

$$
x(t)=\frac{1}{\sqrt{T}} \sum_{k=0}^{K-1} b_{k} e^{j \frac{2 \pi}{T} k t}, 0 \leq t \leq T+T_{G},
$$

where $b=\left[b_{0}, b_{1}, \cdots, b_{K-1}\right]$ denotes data vector of carrier, $T, T_{G}$, and $T+T_{G}$ are the period of fast Fourier transform (FFT) or inverse FFT (IFFT), the length of cyclic prefix and the length of OFDM symbol.

According to the formula (1) and formula (2), the received signal can be expressed as

$$
\begin{aligned}
y(t) & =\frac{1}{\sqrt{T}} \sum_{k=0}^{K-1} b_{k}\left(\sum_{i=0}^{L_{p}-1} a_{i} e^{-j \frac{2 \pi}{T} k \tau_{i}}\right) e^{j \frac{2 \pi}{T} k t}+n(t) \\
& =\frac{1}{\sqrt{T}} \sum_{k=0}^{K-1} b_{k}\left(\sum_{i=0}^{L_{p}-1} a_{i} e^{-j \frac{2 \pi}{T} k \tau_{i}}+n_{k}\right) e^{j \frac{2 \pi}{T} k t},
\end{aligned}
$$

where $n_{k}$ represents the additive white Gaussian noise with the mean of zero and variance of $\sigma^{2}$. The received data at $k$ th carrier $y_{k}$ can be obtained as

$$
y_{k}=b_{k}\left(\sum_{i=0}^{L_{p}-1} a_{i} e^{-j \frac{2 \pi}{T} k \kappa_{i}}+n_{k}\right) \text {. }
$$

Then, multipath channel frequency response at kth carrier can be expressed as

$$
\hat{H}_{k}=\sum_{i=0}^{L_{p}-1} a_{i} e^{-j \frac{2 \pi}{T} k \pi_{i}}+n_{k} \text {. }
$$

The channel frequency response estimation can expressed in vector form

where

$$
\hat{\boldsymbol{H}}=\boldsymbol{H}+\boldsymbol{n}=\boldsymbol{V a}+\boldsymbol{n},
$$

$$
\begin{gathered}
\hat{\boldsymbol{H}}=\left[\begin{array}{llll}
\hat{H}_{0} & \hat{H}_{1} & \cdots & \hat{H}_{K-1}
\end{array}\right]^{T}, \\
\boldsymbol{n}=\left[\begin{array}{llll}
n_{0} & n_{1} & \cdots & n_{K-1}
\end{array}\right]^{T}, \\
\boldsymbol{V}=\left[\begin{array}{llll}
\boldsymbol{v}\left(\tau_{0}\right) & \boldsymbol{v}\left(\tau_{1}\right) & \cdots & \boldsymbol{v}\left(\tau_{K-1}\right)
\end{array}\right]^{T}, \\
\boldsymbol{v}\left(\tau_{i}\right)=\left[\begin{array}{llll}
1 & e^{-j \frac{2 \pi}{T} \tau_{i}} & \cdots & e^{-j \frac{2 \pi}{T}(K-1) \tau_{i}}
\end{array}\right]^{T}, \\
\boldsymbol{a}=\left[\begin{array}{llll}
a_{0} & a_{1} & \cdots & a_{L_{p}-1}
\end{array}\right]^{T} .
\end{gathered}
$$

\section{Root-MUSIC time delay estimation based on propagator method}

Under the hypothesis that the steering matrix $\boldsymbol{V}$ is of full rank, ${ }^{L_{P}}$ rows of $\boldsymbol{V}$ are linearly independent. The other rows can be expressed as a linear combination of these ${ }^{L_{P}}$ rows. We assume that the first ${ }^{L_{P}}$ rows are linearly independent.

\section{Propagator method}

The definition of the propagator is based on the partition of the steering vectors (9) in accordance with

$$
\left.\boldsymbol{V}=\left[\begin{array}{l}
\boldsymbol{V}_{A} \\
\boldsymbol{V}_{B}
\end{array}\right]\right\} \begin{aligned}
& L_{P} \\
& \}-L_{P}
\end{aligned},
$$

where $\boldsymbol{V}_{A}$ and $\boldsymbol{V}_{B}$ are the matrices of dimension $L_{P} \times L_{P}$ and $\left(K-L_{P}\right) \times L_{P}$ respectively.

Owing to non-singular matrix $\boldsymbol{V}_{A}$, the propagator is the unique linear operator $\boldsymbol{P}$ which satisfies with

$$
\boldsymbol{P}^{\mathrm{H}} V_{A}=V_{B} .
$$

The formula (13) is equivalent to

$$
\left[\boldsymbol{P}^{\mathrm{H}},-\boldsymbol{I}_{K-L_{P}}\right] \boldsymbol{V}=\boldsymbol{Q}^{\mathrm{H}} \boldsymbol{V}=\mathbf{0}_{\left(K-L_{P}\right) \times L_{P}},
$$

where $\boldsymbol{I}_{K-L_{P}}$ and $\mathbf{0}_{\left(K-L_{P}\right) \times L_{P}}$ are the identity matrix and the null matrix respectively. 
The autocorrelation matrix $\boldsymbol{R}_{\hat{H} \hat{H}}=E\left[\hat{\boldsymbol{H}} \hat{\boldsymbol{H}}^{\mathrm{H}}\right]$ of $\hat{\boldsymbol{H}}$ can be blocked as

$$
\boldsymbol{R}_{H H}=\left[\begin{array}{ll}
G & F
\end{array}\right],
$$

where $\boldsymbol{G}=\boldsymbol{R}_{\text {Hн }}\left(:, 1: L_{P}\right)$ and $\boldsymbol{F}=\boldsymbol{R}_{\mathrm{HH}}\left(:, L_{P}+1: K\right)$ are the columns from 1 to $L_{P}$ and the columns from $L_{P}+1$ to $K$, respectively. The matrices $\boldsymbol{G}, \boldsymbol{F}$, and $\boldsymbol{P}$ satisfy the following relations

$$
\boldsymbol{G P}=\boldsymbol{F} \text {. }
$$

The least square cost function for solution of (16) can be expressed as

$$
J(\boldsymbol{P})=\|\boldsymbol{F}-\boldsymbol{G P}\|_{F}^{2},
$$

where $\|\cdot\|_{F}$ respresents Frobenius norm.

In consequence, the result of propagator estimation is

$$
\hat{\boldsymbol{P}}=\left(\boldsymbol{G}^{\mathrm{H}} \boldsymbol{G}\right)^{-1} \boldsymbol{G}^{\mathrm{H}} \boldsymbol{F} \text {. }
$$

Both $\boldsymbol{Q}$ and $\boldsymbol{V}$ are orthogonal each other, so the space expanded by $\boldsymbol{Q}$ belongs to the noise subspace. In order to improve the performance, we can introduce a projection operator onto the noise subspace, and substitute $Q$ by its orthonormalized version

$$
\boldsymbol{Q}_{0}=\boldsymbol{Q}\left(\boldsymbol{Q}^{\mathrm{H}} \boldsymbol{Q}\right)^{-1 / 2}
$$

In order to estimate time delay, the pseudospectral function can be obtained as

$$
P_{\mathrm{PM}}(\tau)=\frac{1}{\left|\boldsymbol{v}^{\mathrm{H}}(\tau) \boldsymbol{Q}_{0} \boldsymbol{Q}_{0}^{\mathrm{H}} \boldsymbol{v}(\tau)\right|},
$$

which is similar to the MUSIC algorithm.

\section{Root-MUSIC based on propagator method}

The computational complexity of the MUSIC algorithm is so high as to be difficult to be realized in project, because it need estimate autocorrelation matrix and eigenvalue decomposition. The PM algorithm can obtain the noise subspace by taking advantage of the submatrix of autocorrelation matrix and does not need eigenvalue decomposition for autocorrelation matrix. The spectral peak searching can be made for time delay estimation by the propagator, and the computational complexity of PM is lower than MUSIC algorithm.

We propose a Root-MUSIC time delay estimation based on propagator method which does not need eigenvalue decomposition and spectral peak searching. The algorithm principle is as follows. We can estimate $\boldsymbol{Q}$ by PM algorithm and obtain $\boldsymbol{Q}_{0}$ which can be used as noise subspace by orthogonalization.

According to the rationale of Root-MUSIC algorithm, a polynomial can be defined as

$$
f(z)=\boldsymbol{p}^{\mathrm{H}}(z) \boldsymbol{Q}_{0} \boldsymbol{Q}_{0}^{\mathrm{H}} \boldsymbol{p}(z),
$$

where $z=e^{-j \frac{2 \pi}{\tau} \tau}$.

As long as the roots of the polynomial can be calculated, the time delay can be estimated. It is complicated to solve polynomial, because the polynomial exists $z^{*}$. Therefore, the polynomial (21) can be modified as

$$
f(z)=z^{K-1} \boldsymbol{p}^{\mathrm{T}}\left(z^{-1}\right) \boldsymbol{Q}_{0} \boldsymbol{Q}_{0}^{\mathrm{H}} \boldsymbol{p}(z) .
$$

The time delay estimation can be obtained by the $L_{P}$ roots which are closest to unit circle. The steps of the proposed algorithm can be summarized as step. 1 Calculate the autocorrelation matrix $\boldsymbol{R}_{\hat{H} \hat{H}}$; step. 2 Estimate $\boldsymbol{Q}$ by PM algorithm, and calculate $\boldsymbol{Q}_{0}$ by orthogonalization; step. 3 Find the roots of polynomial (22); step. 4 Obtain the $L_{P}$ roots which are closest to unit circle; step. 5 Estimate $\hat{\tau}_{i}=-\frac{T}{2 \pi}$ angle $\left(\hat{z}_{i}\right), \quad i=0, \cdots, L_{P}-1$.

\section{Simulation results and analysis of computational complexity}

In this section, the simulation results will be discussed for confirming the performance of the 
proposed algorithm, and the analysis of computational complexity will be shown for conforming the lower computational complexity of the proposed algorithm than Root-MUSIC alogrithm.

\section{Simulation results}

We will discuss the performance of super resolution time delay estimation based on RootMUSIC and PM-Root-MUSIC in OFDM system. The number of multipath is 2. The amplitudes of multipath components is 1 and 0.9 respectively. The snapshots is 64, and the number of Monte Carlo simulations is 200. We set the parameters of OFDM system listed in Table 1.

Table 1 OFDM system parameter settings

\begin{tabular}{ccc}
\hline No. & Parameters & Values \\
\hline 1 & Guard duration $T_{G}$ & $1.6 \mu \mathrm{s}$ \\
2 & FFT Period $T_{F F T}$ & $3.2 \mu \mathrm{s}$ \\
3 & System Bandwidth $B$ & $20 \mathrm{MHz}$ \\
4 & The Number of & 64 \\
5 & Subcarrier & $2.4 \mathrm{GHz}$ \\
\hline
\end{tabular}

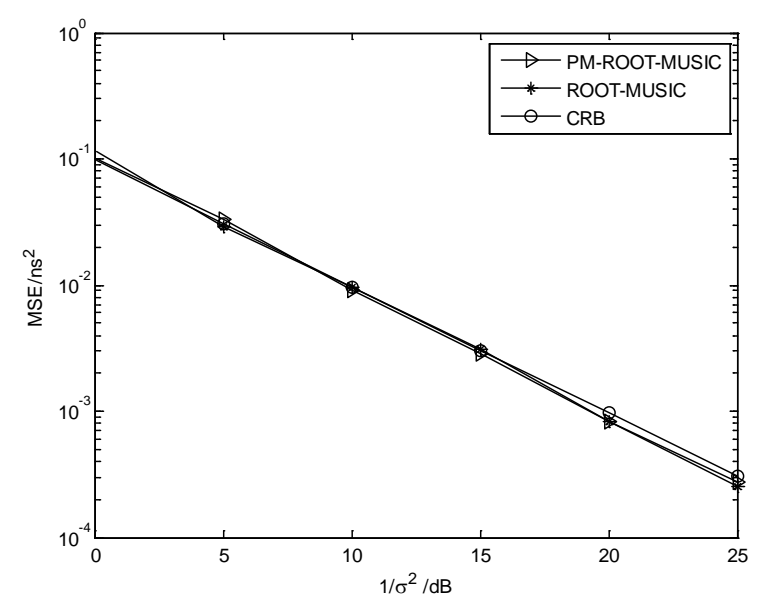

Fig.1: The performance comparison of $1^{\text {st }}$ time delay between these algorithms.

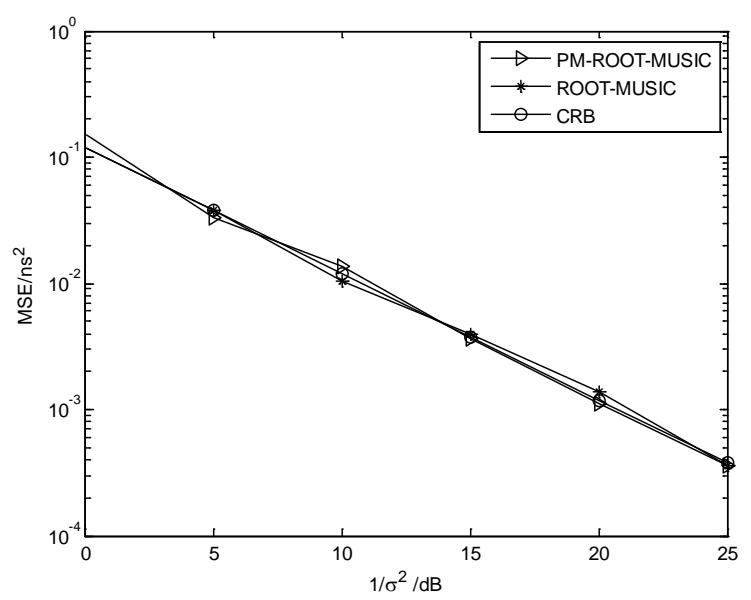

Fig.2: The performance comparison of $2^{\text {nd }}$ time delay between these algorithms.

The performance comparisons between these algorithms are shown in Fig.1 and Fig.2. The mean square error of the Root-MUSIC algorithm and the PM-Root-MUSIC algorithm decrease with increase of the power of noise. The performance of the PM-Root-MUSIC algorithm is close to the Root-MUSIC algorithm, and approaches Cramer-Rao bound (CRB).

\section{Analysis of computational complexity}

The computational complexity of PM algorithm, Root-MUSIC algorithm, and the proposed 
algorithm will be compared below. These algorithms need estimate the the autocorrelation matrix $\boldsymbol{R}_{\hat{H} \hat{H}}$ of $\hat{\boldsymbol{H}}$, whose computational complexity is $O\left(M K^{2}\right)$ times multiplication of complex number. $M$ is the snapshots. The computational complexity of eigenvalue decomposition for Root-MUSIC algorithm $O\left(K^{3}\right)$ times multiplication of complex number. The PM algorithm does not need eigenvalue decomposition, and the computational complexity of orthogonalization and spectral peak searching are $O\left(\left(K-L_{P}\right) L_{P}^{2}\right)$ and $O\left(K L_{P} m_{\tau}\right)$, where $m_{\tau}$ is the number of searching. The RootMUSIC algorithm makes use of finding the roots of polynormial in order to substitute spectral peak searching, so the computational complexity significantly reduces. The proposed algorithm does not need eigenvalue decomposition and spectral peak searching. So the computational complexity of the proposed algorithm significantly reduces.

\section{Conclusions}

The performance of time delay estimation is vital for the evaluation of performance of positioning system. The super resolution has great performance in time delay estimation. However, the computational complexity of super resolution is very high. To address this issue, a new RootMUSIC time delay estimation based on propagator method is proposed. The proposed algorithm does not need eigenvalue decomposition and spectral peak searching. The performance of the algorithm is close to the Root-MUSIC and approaches CRB, and the computational complexity is lower than Root-MUSIC. Simulation results and analysis of computational complexity are conducted to confirm the effectiveness of the proposed algorithm.

\section{References}

[1] Song H. B., Wang H.-G., Hong K., \& Wang L., A novel source localization scheme based on unitary esprit and city electronic maps in urban environments. Progress In Electromagnetics Research, 94, pp.243-262, 2009.

[2] Liu H.-Q., So H.-C., Lui K. W. K., \& Chan F. K. W., Sensor selection for target tracking in sensor networks. Progress In Electromagnetics Research, 95, pp.267-282, 2009.

[3] Liu H.-Q. \& So H.-C., Target tracking with line-of-sight identification in sensor networks under unknown measurement noises. Progress In Electromagnetics Research, 97, pp.373-389, 2009.

[4] Mitilineos S. A. \& Thomopoulos S. C. A., Positioning accuracy enhancement using error modeling via a polynomial approximation approach. Progress In Electromagnetics Research, 102, pp.49-64, 2010.

[5] Zhang W., Hoorfar A., \& Li L., Through-the-wall target localization with time reversal music method. Progress In Electromagnetics Research, 106, pp.75-89, 2010.

[6] Mitilineos S. A., Kyriazanos D. M., Segou O. E., Goufas J. N., \& Thomopoulos S. C. A., Indoor localisation with wireless sensor networks. Progress In Electromagnetics Research, 109, pp.441-474, 2010.

[7] Li X., \& Pahlavan K., Super-resolution TOA estimation with diversity for indoor geolocation. IEEE Trans. Wireless Commun., 3(1), pp.224-234, 2004.

[8] Li X., Ma X., Yan S., \& Hou C., Super-resolution time delay estimation for narrowband signal. IET Radar Sonar \& Navigation, 6(8), pp.781-787, 2012.

[9] Zhang X., Feng G., \& Xu D., Blind direction of angle and time delay estimation algorithm for uniform linear array employing multi-invariance music. Progress In Electromagnetics Research Letters, 13, pp.11-20, 2010.

[10]Oh D., Kim S., Yoon S. H., \& Chong J. W., Two-dimensional ESPRIT-like shift-invariant TOA estimation algorithm using multi-band chirp signals robust to carrier frequency offset. IEEE Trans. Wireless Commun., 12(7), pp.3130-3139, 2013. 
[11]Wang F. Q., Zhang X. F., \& Wang F., Root-MUSIC-based joint TOA and DOA estimation in IR-UWB. Journal on Communications, 35(2), pp.137-145, 2014.

[12]Wang F. Q., \& Zhang X. F., Improved propagator method-based joint TOA and DOA estimation in impulse radio ultra wideband. Journal of Electronics \& Information Technology, 35(12), pp.2954-2959, 2013. 